AEROSPACE REPORT NO. ATR-93(7251)-1

$N A G W-2126$

\title{
Analytic Model of Aurorally Coupled Magnetospheric and Ionospheric Electrostatic Potentials
}

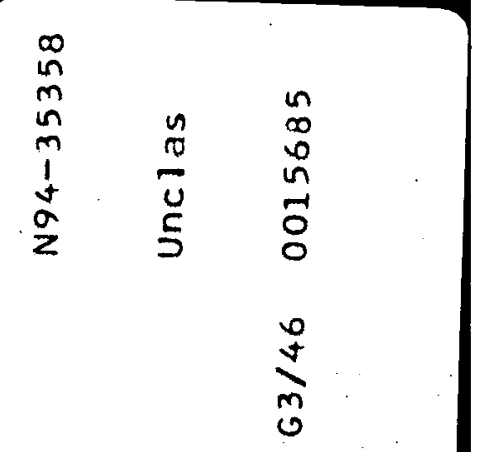

\author{
15 January 1994 \\ NASA-CR-196130 \\ 19940030852
}

Prepared by

J. M. CORNWALL

Space and Environment Technology Center

㟔 0

Technology Operations

Prepared for

NASA

Goddard Space Flight Center

Greenbelt, MD 20771

Grant No. NAGW-2126

Engineering and Technology Group

PUBLIC RELEASE IS AUTHORIZED

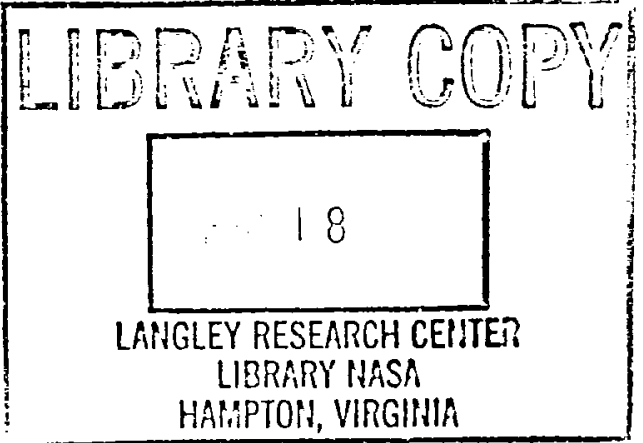




\section{TECHNOLOGY OPERATIONS}

The Aerospace Corporation functions as an "architect-engineer" for national security programs, specializing in advanced military space systems. The Corporation's Technology Operations supports the effective and timely development and operation of national security systems through scientific research and the application of advanced technology. Vital to the success of the Corporation is the technical staff's wide-ranging expertise and its ability to stay abreast of new technological developments and program support issues associated with rapidly evolving space systems. Contributing capabilities are provided by these individual Technology Centers:

Electronics Technology Center: Microelectronics, solid-state device physics, VLSI reliability, compound semiconductors, radiation hardening, data storage technologies, infrared detector devices and testing; electro-optics, quantum electronics, solid-state lasers, optical propagation and communications; $\mathrm{cw}$ and pulsed chemical laser development, optical resonators, beam control, atmospheric propagation, and laser effects and countermeasures; atomic frequency standards, applied laser spectroscopy, laser chemistry, laser optoelectronics, phase conjugation and coherent imaging, solar cell physics, battery electrochemistry, battery testing and evaluation.

Mechanics and Materials Technology Center: Evaluation and characterization of new materials: metals, alloys, ceramics, polymers and their composites, and new forms of carbon; development and analysis of thin films and deposition techniques; nondestructive evaluation, component failure analysis and reliability; fracture mechanics and stress corrosion; development and evaluation of hardened components; analysis and evaluation of materials at cryogenic and elevated temperatures; launch vehicle and reentry fluid mechanics, heat transfer and flight dynamics; chemical and electric propulsion; spacecraft structural mechanics, spacecraft survivability and vulnerability assessment; contamination, thermal and structural control; high temperature thermomechanics, gas kinetics and radiation; lubrication and surface phenomena.

Space and Environment Technology Center: Magnetospheric, auroral and cosmic ray physics, wave-particle interactions, magnetospheric plasma waves; atmospheric and ionospheric physics, density and composition of the upper atmosphere, remote sensing using atmospheric radiation; solar physics, infrared astronomy, infrared signature analysis; effects of solar activity, magnetic storms and nuclear explosions on the earth's atmosphere, ionosphere and magnetosphere; effects of electromagnetic and particulate radiations on space systems; space instrumentation; propellant chemistry, chemical dynamics, environmental chemistry, trace detection; atmospheric chemical reactions, atmospheric optics, light scattering, state-specific chemical reactions and radiative signatures of missile plumes, and sensor out-offield-of-view rejection. 
- DISPLAY $94 \mathrm{~N} 35358 / 2$

94N35358*\# ISSUE 11 CATEGORY 46

RPT\#: NASA-CR-196130 NAS 1.26:196130 ATR-93(7251)-1 CNT\#: NAGW-2126

$94 / 01 / 1512$ PAGES UNCLASSIFIED DOCÚMENT

UTTL: Analytic model of aurorally coupled magnetospheric and ionospheric electrostatic potentials

AUTH: A/CORNWALL, J. M.

CORP: Aerospace Corp., El Segundo, CA. CSS: (Engineering and Technology Group. )

SAP: Avail: CASI HC A03/MF A01

CIO: UNITED STATES

MAJS: /*AURORAL ARCS/*AURORAL ZONES/*AURORAS/*ELECTROSTATICS/*IONIZATION/*POLAR REGIONS

MINS: / ANALYSIS (MATHEMATICS)/ DISSIPATION/ MATHEMATICAL MODELS/ NONLINEARITY/ PHENOMENOLOGY

ABA: Author (revised)

ABS: This paper describes modest but significant improvements on earlier studies of electrostatic potential structure in the auroral region using the adiabatic auroral arc model. This model has crucial nonlinearities (connected, for example. with aurorally produced ionization) which have hampered analysis; earlier work has either been linear, which I will show is a poor approximation or, if nonlinear, either numerical or too ENTER :

specialized to study parametric dependencies. With certain simplifying

MORE 


\section{- DISPLAY 94 N35358/2}

assumptions I find new analytic nonlinear solutions fully exhibiting the parametric dependence of potentials on magnetospheric (e.g.. cross-tail potential) and ionospheric (e.g., recombination rate) parameters. No purely phenomenological parameters are introduced. The results are in reasonable agreement with observed average auroral potential drops, inverted-V scale sizes, and dissipation rates. The dissipation rate is quite comparable to tail energization and transport rates and should have a major effect on tail and magnetospheric dynamics. This paper gives various relations between the cross-tail potential and auroral parameters (e.g., total parallel currents and potential drops) which can be studied ENTER : with existing data sets. 
Aerospace Report No.

ATR-93(7251)-1

\title{
ANALYTIC MODEL OF AURORALLY COUPLED MAGNETOSPHERIC AND IONOSPHERIC ELECTROSTATIC POTENTIALS
}

\author{
Prepared by \\ J. M. Cornwall \\ Space and Environment Technology Center \\ Technology Operations
}

15 January 1994

Engineering and Technology Group THE AEROSPACE CORPORATION

El Segundo, CA 90245-4691

Prepared for

NASA

Goddard Space Flight Center

Greenbelt, MD 20771

Grant No. NAGW-2126

PUBLIC RELEASE IS AUTHORIZED 
Aerospace Report No.

ATR-93(7251)-1

\section{ANALYTIC MODEL OF AURORALLY COUPLED MAGNETOSPHERIC AND IONOSPHERIC \\ ELECTROSTATIC POTENTIALS}

Prepared

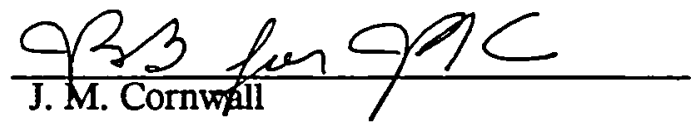

Approved

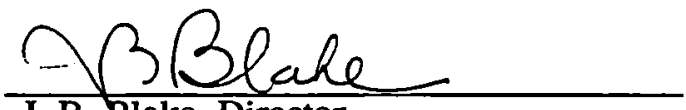

J. B. Blake, Director

Space Sciences Department

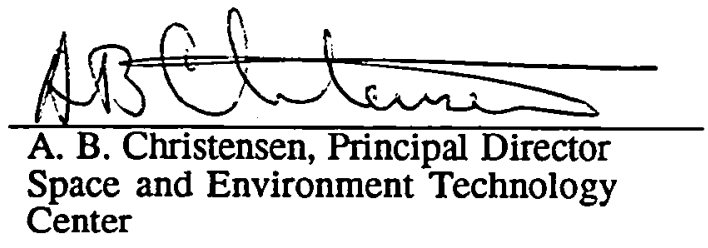




\section{NOTE}

The material reproduced in this report originally appeared in Journal of Geophysical Research. The ATR is published to document the work for the corporate record. 


\title{
Analytic Model of Aurorally Coupled Magnetospheric and Ionospheric Electrostatic Potentials
}

\author{
JOHN M. CORNWALL ${ }^{1}$ \\ Space and Environment Technology Center, The Aerospace Corporation, El Segundo, California \\ This paper describes modest but significant improvements on earlier studies of electrostatic \\ potential structure in the auroral region, using the adiabatic auroral are model. This model has crucial \\ nonlinearities (connected, for example, with aurorally produced ionization) which have hampered \\ analysis; earlier work has either been linear, which I will show is a poor approximation or, if nonlinear, \\ either numerical or too specialized to study parametric dependencies. With certain simplifying \\ assumptions I find new analytic nonlinear solutions fully exhibiting the parametric dependence of \\ potentials on magnetospheric (e.g., cross-tail potential) and ionospheric (e.g., recombination rate) \\ parameters. No purely phenomenological parameters are introduced. The results are in reasonable \\ agreement with observed average auroral potential drops, inverted-V scale sizes, and dissipation rates. \\ The dissipation rate is quite comparable to tail energization and transport rates and should have a \\ major effect on tail and magnetospheric dynamics. This paper gives various relations between the \\ cross-tail potential and auroral parameters (e.g., total parallel currents and potential drops) which can \\ be studied with existing data sets.
}

\section{INTRODUCTION}

In this paper I look again at some issues raised over the years concerning the adiabatic auroral arc model [Chiu and Schulz, 1978; Lyons, 1980, 1981; Chiu and Cornwall, 1980; Chiu et al., 1981]. The essence of this mode is a linear relation between the auroral parallel current $J_{\|}$and the potential drop $\psi \equiv \psi_{i}-\psi_{e}$ between the ionospheric electrostatic potential $\psi_{i}$ and the equatorial potential $\psi_{e}$ along an auroral field line (see equation (2) in section 2). In principle, this model can account for all the gross features (e.g., potential drops, auroral size scales, and dissipation rates) of magnetosphere-ionosphere coupling as governed by auroral phenomena, with no adjustable phenomenological parameters or fitting factors, as was pointed out by the author some years ago [Cornwall, 1983]. However, because the auroral model is nonlinear beyond the current-potential relation, it has not yet been possible to give, even in an idealized model, a precise analytic picture of how potential drops and so forth depend on physically determinate parameters such as the polar cap potential drop, $\psi_{p c}$ and the ionospheric recombination rate constant, $\alpha$. The studies referred to above were either linear or treated nonlinearities numerically for the most part (although Cornwall [1983] did give some nonlinear scaling laws which will be refined here).

In later studies [Cornwall, 1988, 1990], certain exact solutions were found to the nonlinear model equations, but these were too specialized to allow a study of parameter dependence; typically, some combination of independent parameters had to be fixed to allow for a solution. The present investigation is carried out in a similar spirit but with different assumptions and results, which allow most of the interesting physical parameters to be varied freely. In particular, I model the equatorial potential $\psi_{e}$ as Vasyliunas

\footnotetext{
'Permanently at Department of Physics, University of California Los Angeles, California.

Copyright 1993 by the American Geophysical Union.

Paper number 93JA01189. 0148-0227/93/93JA-01189\$05.00
}

[1970] did, which simplifies the nonlinear structure at the price of introducing unphysical discontinuities on the boundary between open and closed field lines. These discontinuities are of little importance to my major results; their primary effect is to make unreliable the detailed shape of $\psi_{i}-\psi_{e}$ very near the central auroral field line.

For present purposes one essential nonlinearity of the adiabatic auroral arc model is associated with a densitydependent Pedersen conductivity. (This and other nonlinearities contribute on smaller size scales to auroral structure and instabilities [Cornwall, 1990; Keskinen et al., 1992], which will not concern us here.) It is a strong nonlinearity in the sense that the ionospheric plasma density on the central auroral line can be 10 or more times greater than the unperturbed density. It can, of course, be dealt with numerically, as the early studies referred to previously in this section did, but that is not my purpose here. We will find that it significantly affects the gross size of auroras, increasing their width by a factor of several over those of a linearized analysis [e.g., Chiu et al., 1981; Cornwall, 1983] to a value of several hundred kilometers. A second nonlinearity arises in the relation between the auroral potential drop $\psi$ and the height-integrated ionospheric plasma density, $N$, which results in nonlinear relations between $\psi$ and $\psi_{p c}$ and between the dissipated auroral power $P$ and $\psi_{p c}$ (the appropriate scaling laws were given earlier by Cornwall [1983]).

Our major results on the relations between the auroral size, $\psi, P$, and $\psi_{p c}$ are given in equations (43)-(49); with no adjustable parameters, they give values within a factor of 2 or better of observed values for typical values of $\psi_{p c}$.

These results have interest not only in themselves but also for the future studies they suggest. Section 1 concludes with some remarks in this direction, setting the present work in a larger context involving tail and magnetospheric convection dynamics.

The standard picture of magnetospheric convection is largely based on the work of Vasyliunas [1970] (see also Fejer [1964]; Schield et al. [1969] for important precursors). In this picture, magnetic field lines are equipotentials, and the two-dimensional electrostatic potential, mapped to the 
ionosphere, is governed by ionospheric current conservation. The effective height-integrated conductivities receive contributions both from the ionosphere and from the ring current, and there are parallel currents driven by the convection discontinuity at the boundary between open and closed field lines. This picture has been modeled with considerable success in computer simulations [e.g., Wolf, 1970; Wolf et al., 1991].

Of course, field lines in the auroral zone are not equipotentials, as many studies have shown [Evans, 1974; Mizera and Fennell, 1977; Reiff et al., 1988; Lindqvist and Marklund, 1990]. The convection modelers have not yet added the physics discussed in this paper to their codes, in part because it adds substantial complexity and, perhaps, in part because it seems at first glance to have impact localized to the auroral zone.

I suggest that the analytic treatment of this paper could be used, at least at first, as a substitute for adding complex codes to the convection models. My results could be used as phenomenological input to existing model codes. I further suggest that some treatment of auroral dissipation will be essential, if the modelers are ever to hope to capture the main features of tail and magnetospheric dynamics, even in regions remote from the auroral zone. The reason is that auroral-ionospheric Joule dissipation is [Cornwall, 1983] comparable to energization and transport rates in the tail and ring current and so plays an important role in global energy balance. (A similar point was made by W. Liu, as cited by Huang et al. [1989].)

Consider the following simple argument. The energy per unit ionospheric area stored in a dipolar flux tube is $\sim L^{4} R_{E} P$, where $R_{E}$ is the radius of the Earth and $P \sim 4 \times$ $10^{-9}$ ergs $\mathrm{cm}^{-3}$ is a typical plasma sheet pressure, while the Joule dissipation rate $\Sigma_{p} E_{\perp}^{2}$ is of the order of $10 \mathrm{ergs} \mathrm{cm}^{-2}$ $s$ or more during an aurora. So the time scale $\tau$ on which an aurora could drain this flux tube of energy is

$$
\tau \approx \frac{L^{4} R_{E} P}{\Sigma_{p} E_{\perp}^{2}} \sim 10^{2}-10^{3} \mathrm{~s}
$$

not large compared to tail transport and energization times. (Note that the tail energy is stored mostly in ions, while the aurora transfers energy from the magnetosphere to the ionosphere mostly via energetic electrons.) It has been long known [Erickson and Wolf, 1980; Schindler and Birn, 1982] that there are serious difficulties in creating a steady state loss-free model of tail convection. This suggested the possibility of nonsteady convection as discussed by the above authors or lossy convection (as indicated, for example, by an effective adiabatic index of $\gamma<1$ [Spence et al., 1987]). An important contributor to loss is cross-tail drift [Kivelson and Spence, 1988; Spence and Kivelson, 1990], and equation (1) suggests that ionospheric Joule dissipation may be just as important.

I am currently in the process of constructing semianalytic models of the effect of auroral dissipation on tail dynamics, which may be of some use. Ultimately, I hope that the computer modelers will take over and vastly improve my simple efforts.

\section{Finding the Electrostatic Potential}

It is well known [Vasyliunas, 1970] that the usual sort of two-dimensional electrostatic potential in and near the polar cap calls for nonvanishing $J_{\|}$, driven by a nonvanishing divergence of horizontal ionospheric currents. Later, Cornwall [1983] identified these parallel currents with auroral currents according to the standard prescription of the adiabatic auroral model:

$$
J_{\|}=-Q\left(\psi_{i}-\psi_{e}\right) \equiv-Q \psi
$$

where $\psi_{i, e}$ is the ionospheric or equatorial potential along a given field line and $Q$ is a parameter of the order of $n e^{2}\left(m_{e} \bar{V}_{e}\right)^{-1}$ with $n$ as the plasma sheet electron density and $\bar{V}_{e}$ as a characteristic plasma sheet electron velocity. This led to the prediction [Cornwall, 1983] that

$$
\psi \sim(4-6) \frac{\Lambda \psi_{p c}}{R_{E}}
$$

where

$$
\Lambda=\left(\Sigma_{p} / Q\right)^{1 / 2} \sim 100 \mathrm{~km}
$$

is the characteristic length scale of the adiabatic auroral arc model and $\psi_{p c}$ is the polar cap potential drop. (Actually, (2) holds only when $\psi_{p c}$ is large enough to give $\psi \geq 1 \mathrm{kV}$; for smaller $\psi$ the necessary $J_{\|}$can be furnished by other means).

The earlier work was largely linear, and even the linear analysis was not carried to completion. In this section I will give the full linear analysis and also an exact nonlinear solution to the model equations. In some respects the linear analysis is not a good approximation to the exact solution (it has the wrong spatial scale), but it can be plausibly fudged to give global results (e.g., the central potential drop) quite similar to the exact analysis. Moreover, it illustrates how I turn the equatorial potential $\psi_{e}$, which appears as a source term in the nonlinear equations, into a boundary condition.

The two fundamental equations of the adiabatic auroral arc model are

$$
\begin{gathered}
\nabla_{\perp} \cdot\left(\Sigma \cdot \nabla_{\perp} \psi_{i}\right)=-J_{\|}=Q \psi \\
\frac{\partial N}{\partial t}+V_{E} \cdot \nabla_{\perp} N=\frac{\Gamma}{e} Q \psi-\alpha\left(N^{2}-N_{o}^{2}\right)
\end{gathered}
$$

where $N$ is the height-integrated ionospheric plasma density, $\Sigma$ is the height-integrated ionospheric conductivity tensor, $\Gamma$ is the number of electron-ion pairs produced per incident auroral electron, $\alpha$ is a height-integrated dissociative recombination coefficient, $\alpha N_{o}^{2}$ summarizes nonauroral sources of ionospheric ionization, and $V_{E}$ is the electric drift velocity such that

$$
V_{E}=\frac{-c}{B^{2}}(\nabla \psi \times B) .
$$

The return current region lying just outside the auroral region is governed by different physics, which I will not attempt to model in detail here (for an earlier attempt, see Chiu et al. [1981]). Presumably, $\psi-\psi_{e}$ goes through zero at the edges of the aurora and then becomes slightly negative in the return current region, but with $\left|\psi-\psi_{e}\right|$ much smaller than in the auroral region. In the simplified analysis I will give, $\psi-\psi_{e}$ approaches zero only at asymptotically large distances from the zero, and so the retum current is also far away. I emphasize that this is a mere artifact, which unless it were mistakenly taken literally, has no effect on our conclusions. In reality, the return current region lies just adjacent to the auroral region, only a few auroral scale lengths, $\Lambda$, away. 
Equations (5-7) contain a vast number of effects which are readily studied with a computer but whose analytic treatment is either difficult or impossible. These include various instabilities, as well as nonlinearities, the primary effect of which is to increase the density $N$ and conductivity $\Sigma$ in the auroral region. This conductivity enhancement has a number of important and well-known consequences for convection, which will be studied elsewhere in connection with auroralmagnetospheric coupling. For now, I want to find an analytically treatable treatment of this conductivity nonlinearity in the spirit of earlier [Cornwall, 1988, 1990] analytic solutions to similar auroral problems. These earlier solutions are not useful in the present context, because they do not allow for a priori specification of the input driving potential $\psi_{e}$. Fortunately, $\psi_{e}$ is not completely arbitrary, and progress can be made by modeling $\psi_{e}$ as Vasyliunas [1970] and Cornwall [1983] did; $\psi_{e}$ consists of two separate solutions to Laplace's equation (reflecting charge neutrality), joined on a closed contour representing the auroral zone and continuous across this contour. However, $\nabla \psi_{e}$ is discontinuous across the contour. This discontinuity leads to $J_{\|}$proportional to a Dirac delta function. The input potential $\psi_{e}$ then only appears as a boundary condition on a homogeneous nonlinear equation, some special solutions to which can be found analytically.

Now for the details. I begin by simplifying the ion conductivity equation (6), dropping the terms on the left-hand side. These terms are primarily important [Cornwall, 1990; Keskinen et al., 1992] for auroral instabilities on time scales of $\sim 100 \mathrm{~s}$, but on longer time scales it is a reasonable approximation to assume rough equilibrium between recombination and auroral precipitation. Then (6) yields

$$
\psi \equiv \psi_{i}-\psi_{e}=\frac{\alpha e}{\Gamma Q}\left(N^{2}-N_{o}^{2}\right)
$$

and (5) is rewritten in terms of $\psi$ :

$$
\nabla_{\perp} \cdot\left(\Sigma \cdot \nabla_{\perp} \psi\right)-Q \psi=-\nabla_{\perp} \cdot\left(\Sigma \cdot \nabla_{\perp} \psi_{e}\right)
$$

Under the assumption that the various parameters $(\alpha, \Gamma$, $N_{o}$ ) which appear in (8) are constants, the Hall term drops out of the left-hand side of (9), since then both $\Sigma$ and $\psi$ are functions of the single function $N$. Thus (9) becomes

$$
\nabla_{\perp} \cdot\left(\Sigma_{p} \nabla_{\perp} \psi\right)-Q \psi=-\nabla_{\perp} \cdot\left(\Sigma \cdot \nabla_{\perp} \psi_{e}\right) .
$$

Now we need to specify the source $\psi_{e}$ and the right-hand side of $(10)$. In the equipotential case $(\psi=0)$ the right-hand side vanishes almost everywhere except on the convection boundary between open and closed lines, where it is a delta function. I give a simple example of such a source term, based on constant $\Sigma$ and $\psi_{e}$ as two different solutions of Laplace's equation, joined at the convection boundary. Use spherical coordinates with the magnetic dipole axis as the $z$ axis and the azimuthal angle $\phi$ defined to be $\pi / 2$ at dawn and $-\pi / 2$ at dusk; $\theta$ is the magnetic colatitude. Let

$$
\begin{gathered}
\psi_{c}=\frac{1}{2} \psi_{p c} \frac{\left(\tan \frac{1}{2} \theta\right) \sin \phi}{\tan \frac{1}{2} \theta_{p c}} \quad \theta<\theta_{p c} \\
\psi_{e}=\frac{1}{2} \psi_{p c} \frac{\sin \psi_{p c} \sin \phi}{\sin \theta} \pi-\theta_{p c}>\theta>\theta_{p c} .
\end{gathered}
$$

(For the southern polar cap, replace $\theta$ with $\pi-\theta$ and $\tan \frac{1}{2} \theta_{p c}$ with $\cot \frac{1}{2} \theta_{p c}$ in (11). Here $\psi_{p c}$ is the dawn-to-dusk potential across the polar cap, and the convection boundary is at $\theta=\theta_{p c}$ (or $\theta=\pi-\theta_{p c}$ in the south). Both (11) and (12) satisfy Laplace's equation in the angular variables. The form (11) represents an essentially constant dawn-dusk electric field over the polar cap, as one sees from the stereographic projection of the unit sphere from the south pole to the $x-y$ plane tangent to the north pole, with

$$
\begin{aligned}
\tan \frac{1}{2} \theta \rightarrow \rho ; x & =\rho \cos \phi \\
\phi \rightarrow \phi ; y & =\rho \sin \phi .
\end{aligned}
$$

Then (11), in the projected variables, is $\psi \sim y$, yielding a constant field.

I will now make the idealization in (10) of saving only the most singular term, which is a delta function. It is this which allows further progress to be made, since the nonlinear terms on the left-hand side of $(10)$ depend on $\psi_{e}$ only through boundary conditions at $\theta=\theta_{p c}, \pi-\theta_{p c}$. The result is that (10) becomes, in the northern hemisphere,

$$
\begin{aligned}
\nabla_{\perp} \cdot\left(\Sigma_{p} \nabla_{\perp} \psi\right)-Q \psi= & \frac{\psi_{p c} \Sigma_{p} \sin \phi}{2 R_{E}^{2} \sin \theta} \\
& \cdot(1+\cos \theta) \delta\left(\theta-\theta_{p c}\right),
\end{aligned}
$$

where, on the right-hand side, everything depending on $\theta$ (including $\Sigma_{p}$ ) is to be evaluated at $\theta=\theta_{p c}$. I will not explicitly write out the contributions from the southern hemisphere, which are easily supplied by symmetry.

Note that in the idealization of saving only the most singular source term, the specific forms (11) and (12) for $\psi_{e}$ and the assumption of constant $\Sigma$ when $\theta \neq \theta_{p c}$ are irrelevant; all that matters is the coefficient of the delta function in (14).

To find a solution to (14) two solutions to the homogeneous version of this equation must be found, matched in value at $\theta=\theta_{p c}$, and the discontinuity in $\theta$ derivatives adjusted to match the delta function. The two solutions are chosen so that each decays exponentially with angular distance from $\theta_{p c}$. Far from $\theta=\theta_{p c}$ the conductivity $\Sigma_{p}$ approaches, it is assumed, a constant value, at least in the sense that $\Sigma_{p}$ varies more slowly (owing, for example, to day-night effects) than it does due to precipitation in the auroral zone. In that case, (14) is a linear equation, straightforwardly solved. Later in this section the linear version of (14) is solved, including the delta function source; this both yields the necessary linear solutions far from the auroral zone and illustrates the matching procedure used to accommodate the delta function.

\subsection{Linear Case}

The equation to be solved for constant $\Sigma_{p}$ is

$$
\begin{aligned}
\frac{1}{\sin \theta} \frac{\partial}{\partial \theta}\left(\sin \theta \frac{\partial \psi}{\partial \theta}\right)+\frac{1}{\sin ^{2} \theta} & \frac{\partial^{2} \psi}{\sin \theta}-\lambda^{2} \\
= & K \frac{\sin \phi}{\sin \theta} \delta\left(\theta-\theta_{p c}\right),
\end{aligned}
$$

where 


$$
\begin{aligned}
& K=\frac{1}{2} \psi_{p c}\left(1+\cos \theta_{p c}\right) \\
& \lambda=R_{E} \Lambda=R_{E}\left(Q / \Sigma_{p}\right)^{1 / 2} .
\end{aligned}
$$

Since $\Lambda$, the width of the (idealized) auroral zone, is about $100 \mathrm{~km}, \lambda \gg 1$. We seek a solution of the form

$$
\psi=K \sin \phi G\left(\theta, \theta_{p c}\right),
$$

where $G\left(\theta, \theta^{\prime}\right)$ is a Green's function for Legendre's equation with $m=1, l(l+1)=-\lambda^{2}$. That is,

$$
l=-\frac{1}{2} \pm \frac{1}{2}\left(1-4 \lambda^{2}\right)^{1 / 2} \simeq-\frac{1}{2} \pm i \lambda .
$$

In the limit of large $\lambda$ the exact solution for $G$ can be formally expressed as a linear combination of the conical functions $P_{-1 / 2+i \lambda}^{1}(\cos \theta), Q_{-1 / 2+i \lambda}^{1}(\cos \theta)$ [e.g., Abramowitz and Stegun, 1964]. However, it is difficult to find useful forms for these rather obscure special functions, and we choose instead to give an approximate solution in terms of the well-known functions $K_{1}, I_{1}$ (Hankel and Bessel functions of imaginary argument). For small $\theta$ the homogeneous solutions to (15) are $K_{1}(\lambda \theta)$ and $I_{1}(\lambda \theta)$, valid when $\lambda \theta^{3} \ll 1$. Since $\lambda \theta^{3} \gg 1$, these do not cover a useful range in $\theta$. A better approach is to seek homogeneous solutions of the form, for example, $F(\theta) K_{1}[\lambda R(\theta)]$, determining $F$ and $R$ so that dangerous terms in the differential equation proportional to $\lambda$ or $\lambda^{2}$ are exactly cancelled. A straightforward computation shows that this requires

$$
R=\theta, F=\left(\frac{\theta}{\sin \theta}\right)^{1 / 2}
$$

With this choice the fractional derivation of $F K_{1}(\lambda R)$ from solving (15) is $\theta^{2} / 36$ for small $\theta$ and $O\left(\lambda^{-2}\right)$ for large $\theta$.

It is easy to find the appropriate linear combinations to match the delta function in (15), with the final result that the solution to the linear problem of (15) is

$$
\begin{gathered}
\psi=-\frac{K \sin \phi}{2}\left(\frac{\theta \theta_{p c}}{\sin \theta \sin \theta_{p c}}\right)^{1 / 2}\left[K_{1}(\lambda \theta) I_{1}\left(\lambda \theta_{p c}\right) H(\theta\right. \\
\left.\left.-\theta_{p c}\right)+I_{1}(\lambda \theta) K_{1}\left(\lambda \theta_{p c}\right) H\left(\theta_{p c}-\theta\right)\right],
\end{gathered}
$$

where $H(x)$ is the unit step function $(H(x)=1, x>0$; $=$ $0, x>0$ ). This potential falls off exponentially (roughly as $\left.\exp \left[-\lambda\left|\theta-\theta_{p c}\right|\right]\right)$ on both sides of $\theta_{p c}$. Note that $\lambda \theta_{p c} \gg$ 1 , so that in the vicinity of the auroral zone one should use large-argument asymptotic expansions to evaluate the Bessel functions. When this is done, one finds for the potential drop at $\theta=\theta_{p c}, \sin \phi=-1$ :

$$
\psi_{c} \equiv \psi\left(\theta=\theta_{p c}\right)=\frac{\Lambda \psi_{p c}}{4 R_{E} \sin \theta_{p c}} \simeq \Lambda E_{\perp} .
$$

In extending this linear solution to a nonlinear solution the point is that $\Lambda$ depends on $\Sigma_{p}$ and thus on the heightintegrated plasma density $N$, which by (8) depends on $\psi$. A simple-minded approach to this nonlinearity is to use (8) to express $N$ (and $\Sigma_{p}$ ) in terms of $\psi$, and then (22) becomes an algebraic equation for $\psi_{c}$ :

$\psi_{c}=\frac{\sin \phi \psi_{p c}}{4 R_{E} \sin \theta_{p c}}\left(\frac{1}{Q} \frac{\partial \Sigma_{p}}{\partial N}\right)^{1 / 2}\left(N_{o}^{2}+\frac{\Gamma Q \psi_{c}}{\alpha e}\right)^{1 / 4}$.
(In the limit $N_{o} \rightarrow 0$ the scaling law $\psi_{c} \sim \psi_{p c}^{4 / 3}$ was already given by Cornwall [1983].) We will compare this equation later to the nonlinear solution developed next.

\subsection{Nonlinear Case}

The nonlinearities are important only in the immediate vicinity of the auroral zone, which has a small width compared to $R_{E}$. Therefore I will use the flat earth approximation in the equations, with the $x$ coordinate perpendicular to the auroral zone and $y$ along it. The inhomogeneous delta function such as in (14) need not be written explicitly, since its only role is to furnish a boundary condition at the auroral zone. Equation (8) is used to eliminate $\psi$ in favor of $N$, assuming that $N_{0}$ and $\alpha / \Gamma Q$ in this equation are constants. With the observation that $\Sigma_{p}$ is linear in $N$, so that $\partial \Sigma_{p} / \partial N$ is also constant, the homogeneous version of $(10)$ is

$\nabla_{\perp} \cdot\left(N \nabla_{\perp} N^{2}\right)-Q\left(\partial \Sigma_{p} / \partial N\right)^{-1}\left(N^{2}-N_{o}^{2}\right)=0$

or

$$
\begin{gathered}
\nabla_{\perp}^{2} n^{3}-\frac{3}{2 \Lambda_{0}^{2}}\left(n^{2}-1\right)=0 \\
\Lambda_{o}^{2}=\frac{\Sigma_{p o}}{Q}, \Sigma_{p o}=\Sigma_{p}\left(N=N_{o}\right), n=\frac{N}{N_{0}} .
\end{gathered}
$$

Nonlinear equations similar to (24) have been studied before analytically [Cornwall, 1988, 1990] and numerically [Keskinen et al., 1992] with full two-dimensional dependence. However, the known two-dimensional analytic solutions are too restricted for the present purpose, and my present strategy is to save in (24) and (25) only gradients in the $x$ direction (across the aurora, roughly north-south). Thus I am not modeling auroral blobs and transient (i.e., unstable) local structures. In any event, in the absence of structural magnetospheric forcing the gradient structure which is more or less persistent is the $x$ direction gradient which is saved. There are also examples [Cornwall, 1988] of KelvinHelmholtz stable fully two-dimensional structures in which the $x$ and $y$ direction length scales are essentially the same and roughly equal to the scale length I will find.

So I will ignore the $y$ variation and replace (25) with

$$
F^{\prime \prime}-\frac{3}{2} \Lambda_{0}^{-2}\left(F^{2 / 3}-1\right)=0,
$$

where $F=n^{3}$ and prime indicate $\partial / \partial x$. This equation can be reduced to quadratures; I quote the solution in terms of the original variable $n$ :

$$
\int_{n}^{n_{c}} \frac{d n n^{2}}{(n-1)}\left[\left(n+\frac{2}{3}\right)^{3}+\frac{10}{27}\right]^{-1 / 2}= \pm \frac{x}{5^{1 / 2} \Lambda_{o}} .
$$

This integral supplies the boundary conditions that at $x=0$, $n=n_{c} \equiv N_{c} / N_{o}$, where $N_{c}$ is the central ionospheric density in the auroral zone; typically, $n_{c} \gg 1$. Another constant of integration has been supplied so that as $|x| \rightarrow \infty$, $n \rightarrow 1$ at an exponential rate:

$n \underset{x \rightarrow \infty}{\rightarrow} 1+C_{1} \exp \left(-x / \Lambda_{o}\right)+O\left[\exp \left(-2 x / \Lambda_{0}\right)\right]$ 
We will soon see that the constant $C_{1}$ in (29) is determined by $n_{c}$, which is in turn determined by matching a discontinuity in $n^{\prime}(x=0)$ to $J_{\|}$, as in the linear case.

The integral in (28) can be reduced to a combination of integrals of rational functions plus an elliptic integral of the third kind [e.g., Whittaker and Watson, 1952] and so can be said to yield an exact solution in terms of known functions, at least in principle. But this form is of little use, because it gives $x$ in terms of $n$ while we want $n$ in terms of $x$. Nevertheless, for the dedicated reader who wishes to pursue it, I quote the change of variables which yields a standard elliptic integral form:

$$
u=\frac{n+\frac{2}{3}+\frac{1}{3} 10^{1 / 3}\left(1-3^{1 / 2}\right)}{n+\frac{2}{3}+\frac{1}{3} 10^{1 / 3}\left(1+3^{1 / 2}\right)} .
$$

To determine the constant $C_{1}$, one may use (29) to replace $x / \Lambda_{o}$ in (28) to find

$$
\begin{aligned}
\ln C_{1}=\lim _{n \rightarrow 1} 5^{1 / 2} \int_{n}^{n_{e}} \frac{d n n^{2}}{(n-1)}\left[\left(n+\frac{2}{3}\right)^{3}\right. & \left.+\frac{10}{27}\right]^{-1 / 2} \\
& +\ln (n-1) .
\end{aligned}
$$

By adding and subtracting an integral, which can be explicitly done and which removes the singularities in the integrand of (31) at both endpoints, one can find

$$
C_{1}=\frac{4}{5}\left(\frac{\beta-1}{\beta+1}\right) \exp [2(\beta-1)+\gamma]
$$

where

$$
\begin{gathered}
\beta=\left(5 n_{c}-4\right)^{1 / 2} \\
\gamma\left(n_{c}\right)=5^{1 / 2} \int_{1}^{n_{c}} \frac{d n}{n-1}\left\{n ^ { 2 } \left[\left(n+\frac{2}{3}\right)^{3}\right.\right. \\
\left.\left.+\frac{10}{27}\right]^{-1 / 2}-\left(n-\frac{4}{5}\right)^{1 / 2}\right\} \\
=\gamma\left(n_{c}=\infty\right)+\frac{6}{5}\left(5^{1 / 2}\right) n_{c}^{-1 / 2}+O\left(n_{c}^{-1}\right) \\
\gamma_{c}\left(n_{c}=\infty\right)=-2.50
\end{gathered}
$$

where the last form of (34) is useful for large $n_{c}$.

Rather than continue with analyzing the integral (28), one finds a more useful form of the solution for large and small $|x|$ by direct solution of the differential equation. For small $|x|$ this amounts to an expansion in powers of $n_{c}^{-2} \ll 1$ and leads to

$$
\begin{aligned}
n=n_{c}(1 \pm a x)^{2}[1+ & \frac{5}{9} n_{c}^{-2}(1 \pm a x)^{-4} \\
& \left.-\frac{50}{567} n_{c}^{-4}(1 \pm a x)^{-8}+\cdots\right]
\end{aligned}
$$

where

$$
a=\left[2 \Lambda_{o}\left(5 n_{c}\right)^{1 / 2}\right]^{-1}
$$

and the upper sign in (35) is for $x<0$ and the lower sign for $x>0$. This form of the solution is useful out to $a|x| \approx 1$ $-n_{c}^{-1 / 2}$, where $n=O(1)$.

For large $|x|$ a similar analysis leads to

$$
\begin{aligned}
n=1+C_{1} e^{ \pm x / \Lambda_{0}}-(7 / 6)\left[C_{1} \exp \left( \pm x / \Lambda_{0}\right)\right]^{2}+(101 / 48) \\
\cdot\left(C_{1} e^{ \pm x / \Lambda_{0}}\right)^{3}+\cdots
\end{aligned}
$$

with the same sign convention as in (36). Here $C_{1}$ is the same constant as in (32). Note that it is qualitatively (but not necessarily quantitatively) accurate to estimate $C_{1}$ by finding a point $x_{1}$ where the slopes and values of the large $|x|$ and small $|x|$ forms of $n$ in (35)-(37) match. Just saving the first nontrivial terms yields

$$
\begin{gathered}
x_{1}=(20)^{1 / 2} \Lambda_{o}\left(n_{c}^{1 / 2}-C_{2}^{1 / 2}\right) \equiv(20)^{1 / 2}\left(\Lambda_{c}-C_{2}^{1 / 2} \Lambda_{o}\right) \\
C_{1}=\left(C_{2}-1\right) \exp \left(x_{1} / \Lambda_{o}\right) \\
C_{2}=\frac{1}{10}\left(11+21^{1 / 2}\right)=1.56 .
\end{gathered}
$$

It follows that for large $n_{c}$ (either from the above equations or from the more accurate (32)), the transition from the nonlinear regime (40) to the linear regime (saving only the first term of (37)) sets in at a value of $x$ of the order of $5 \Lambda_{c}$, where $\Lambda_{c}$ is the auroral scaling length $\left(\Sigma_{p} / Q\right)^{1 / 2}$ evaluated at the center of the auroral zone. In effect, $x_{1}$ is the distance from the center of the aurora to the unperturbed ionosphere, and is several times larger than one would have supposed from a linear analysis.

The scaling $x_{1}-(20)^{1 / 2} \Lambda_{c}$ is one of several key results following from the nonlinear analysis. The second result is a formula for the central density $N_{c}$ or, equivalently, for $\psi_{c} \equiv$ $\psi(x=0)$ using (8). It is derived as follows: First, the inhomogeneous term for the nonlinear equation (27) is supplied from the fundamental equation (14), using $x=R_{E}\left(\theta_{p c}\right.$ $-\theta$ ) and the relation between $\psi$ and $N$ in (8). The result for the duskside aurora where $\sin \phi=-1$ is

$$
\begin{aligned}
\left(n^{3}\right)^{\prime \prime}-\frac{3}{2 \Lambda_{o}^{2}}\left(n^{2}-1\right)=-\left(\frac{3 \Gamma Q}{2 \alpha e N_{o}^{2} \Sigma_{p o}}\right) \\
\cdot\left(\frac{\psi_{p c} \Sigma_{p c}\left(1+\cos \theta_{p c}\right)}{2 R_{E} \sin \theta_{p c}}\right) \delta(x) \equiv-\Delta \delta(x) .
\end{aligned}
$$

One inserts the solution (35) to find

$$
12 a n_{c}^{3}\left(1+\frac{5}{9} n_{c}^{-2}-\frac{125}{567} n_{c}^{-4}+\cdots\right)=\Delta .
$$

It turns out that $n_{c} \gg 1$, so only the first term of this expansion is saved. Then, using the definition of $a$ in (36) and rearranging, one finds

$$
N_{c}^{3 / 2}=\frac{5^{1 / 2} \Gamma}{8 \alpha e}\left(Q \frac{\partial \Sigma_{p}}{\partial N}\right)^{1 / 2} \frac{\psi_{p c}\left(1+\cos \theta_{p c}\right)}{R_{E} \sin \theta_{p c}} .
$$

Putting in nominal ionospheric numbers $\left(\Gamma=100, \alpha=10^{-14}\right.$ $\mathrm{cm}^{2} \mathrm{sec}^{-1}, Q=0.1 \mathrm{~cm}^{-1} \mathrm{sec}^{-1}$, and $\partial \Sigma_{p} / \partial N=3 \mathrm{~cm}^{3}$ $\sec ^{-1}$, one finds 


$$
N_{c}=3 \times 10^{12} \mathrm{~cm}^{-2}\left(\frac{\psi_{p c}}{60 \mathrm{kV}}\right)^{2 / 3} .
$$

(I use $60 \mathrm{kV}$ as a reference polar cap potential, because a similar value is associated with the threshold for substorm occurrence [see Weimer et al., 1992; Ahn et al., 1992].)

The third key result expresses the central potential drop $\psi_{e}$ in terms of the polar cap potential drop, $Q$, and ionospheric parameters and is just a combination of (8) and (43):

$$
\begin{aligned}
\psi_{c} & =\frac{\alpha e}{\Gamma Q}\left(N_{c}^{2}-N_{o}^{2}\right) \simeq \frac{\alpha e N_{c}^{2}}{\Gamma Q} \\
& =\frac{5^{2 / 3}}{16}\left(\frac{\Gamma}{Q \alpha e}\right)^{1 / 3}\left(\frac{\partial \Sigma_{p}}{\partial N}\right)^{1 / 3}\left[\frac{\psi_{p c}\left(1+\cos \theta_{p c}\right)}{R_{E} \sin \theta_{p c}}\right]^{4 / 3} .
\end{aligned}
$$

Using the same constants as before

$$
\psi_{c}=3.4 \mathrm{kV}\left(\frac{\psi_{p c}}{60 \mathrm{kV}}\right)^{4 / 3}
$$

This result can be compared to the $N_{0}=0$ version of (23), an equation based on a simple-minded fudging of the linear theory. One can easily check that (23) in the $N_{0}=0$ limit gives (45) except that the constant factor $5^{2 / 3}=0.183$ in (45) is replaced by $4^{-4 / 3} \simeq 0.157$. The agreement is remarkably close.

A final key result is the auroral dissipation of power, integrated over the auroral zone. I will define a theoretically useful, if not immediately observationally relevant, excess dissipation power $P$ as that due to the field $E_{\perp}=-\nabla_{\perp} \psi$ associated with the difference $\left(\psi=\psi_{i}-\psi_{e}\right)$ between the ionospheric potential with and without auroras. So

$$
P=2 R_{E} \sin \theta_{p c} \int d x d \phi \Sigma_{p}\left(\nabla_{\perp} \psi\right)^{2}
$$

where the factor of 2 counts both polar caps and the integrals over $d x$ (distance across the aurora) and $d \phi$ extend over the auroral zone. To be definite, I will use for the $x$ dependence of $N$ and $\psi$ the first term in (35), valid for $n_{c} \gg 1$, integrate in $x$ over $-a^{-1}<x<a^{-1}$, assume that $N$ and $\psi$ depend on $\sin \phi$ as their dependence on $\psi_{p c}$ would suggest, that is, $\psi-$ $|\sin \phi|^{4 / 3}$ and $N \sim|\sin \phi|^{2 / 3}$, and integrate $\phi$ over the region $\sin \phi \leq 0$. The result is

$$
\begin{aligned}
& P=\left(\frac{5}{64}\right) \frac{\Gamma\left(\frac{1}{2}\right) \Gamma\left(\frac{11}{3}\right)}{\Gamma\left(\frac{25}{6}\right)}\left(\frac{\Gamma}{\alpha e}\right)\left(\frac{\partial \Sigma_{p}}{\partial N}\right)^{2} \frac{\psi_{p c}^{3}\left(1+\cos \theta_{p c}\right)^{2}}{R_{E}^{2} \sin ^{2} \theta_{p c}} \\
& P=10^{17} \text { ergs } \sec ^{-1}\left(\frac{\psi_{p c}}{60 \mathrm{kV}}\right)^{3} .
\end{aligned}
$$

This is substantial dissipation, quite enough to influence substorm and tail processes as discussed in connection with (1) in section 1 .

I am unaware of any studies in the literature which deal directly with the main results in (44), (45), and (46). But it is clear that the predicted numbers are, in an average sense, in reasonable agreement with auroral observations [e.g., Evans, 1974; Reiff, 1988; Lindqvist and Marklund, 1990; Chiu et al., 1982].

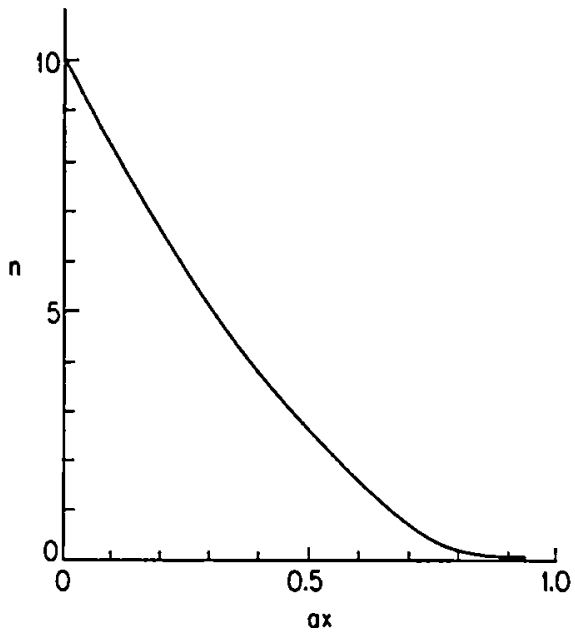

Fig. 1. Scaled density $n=N / N_{o}$ versus scaled length $a x$ (for $a$, see (36)), for $n_{c}=10$.

\section{Concluding Remarks}

My main results are (36), giving the inverted-V scale length $a^{-1}$ as $\sqrt{20} \Lambda_{c}$, where $\Lambda_{c}$ is the linear scale length (see (4)), based on the central Pedersen conductivity, and (43)-(49), giving $N_{c}, \psi_{c}$, and the dissipated power $P$. The scale length is rather bigger than one would have estimated from the linear theory in section 2, and so the linear profiles are not very good approximations. However, the ad hoc equation (23), which grafts certain elements of the nonlinear theory on to the linear analysis, gives a value for $\psi_{c}$ in good agreement with the full nonlinear analysis. This analysis gives values for $N_{c}, \psi_{c}$, and $P$ (with no arbitrary parameters), which are in decent agreement with observed values, and yields scaling laws for these quantities in their dependence on $\psi_{c}$ which can be experimentally studied.

One cannot expect fully realistic spatial profiles of $\psi$ and $N$ near the center of the auroral zone, because my fundamental hypothesis for the magnetospheric potential $\psi_{e}$ (equations (11) and (12)) yields cusps in $\psi$ and $N$ at $\theta=\theta_{p c}$ or $x=0$. These are illustrated in Figures 1 and 2, in which $N$ and $\psi$ are plotted for a typical value of $n_{c}=10$. In principle, $N$ and $\psi$ should approach $x=0$ with zero slope, since these are symmetric around $x=0$. There is no reason to distrust these profiles when the density and potential have dropped by, say, a factor of 2 , and the dissipated power $P$ is an integral over the profiles which is insensitive to the cusp behavior. The cusps will, of course, be removed by a smoother choice of $\psi_{e}$, but precisely how to make this choice is not presently clear.

Having analytic and fully parametrized expressions for, for example, the dissipated power $P$ (sec (48)) will be very useful for theoretical investigations of tail transport and dynamics. As mentioned earlier, this power is comparable to that lost in cross-tail drift, and can have a fundamental impact on the picture of disturbed tail phenomena. Investigations to be reported later are now underway on this subject.

It is, of course, important to know whether the predictions of this paper concerning the relation between $\psi_{p c}$ and various auroral phenomena are borne out by data. The 


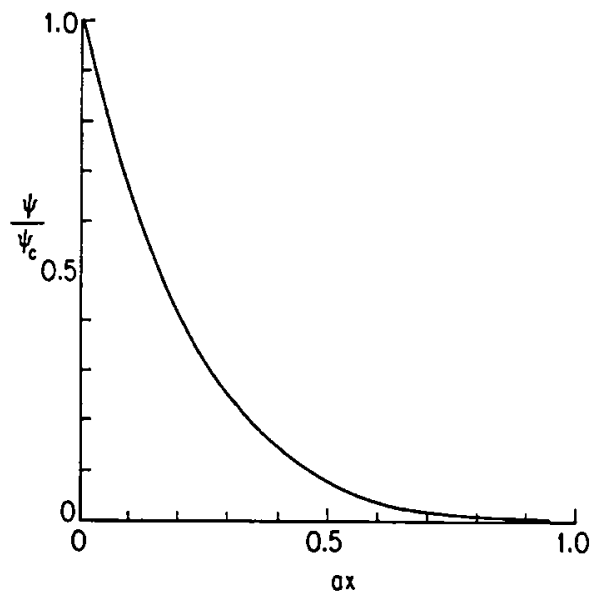

Fig. 2. Ionosphere-equatorial potential difference $\psi$, scaled by the central value $\psi_{e}$ (for which, see (45) and (46)), for $n_{c}=10$.

material necessary for such a study, in the form of convection field data (yielding $\psi_{p c}$ ), field-aligned current data, and field-aligned potential drops, all exists and needs to be correlated. The intervals for which all these data are simultaneously available is by no means negligible. A study of this sort would be of great interest quite aside from the present theoretical considerations.

Acknowledgments. This work was supported by NASA grant NAGW-2126 (Space Physics Theory program) and by the Aerospace-sponsored research program.

The Editor thanks M. J. Keskinen and D. R. Weimer for their assistance in evaluating this paper.

\section{REFERENCES}

Abramowitz, M., and I. A. Stegun, Handbook of Mathematical Functions With Formulas, Graphs, and Mathematical Tables, p. 336, National Bureau of Standards, Washington, D. C., 1964.

Ahn, B. H., Y. Kamide, H. W. Kroehl, and D. J. Gorney, Crosspolar cap potential difference, auroral electrojet indices, and solar wind parameters, J. Geophys. Res., 97, 1345, 1992.

Chiu, Y. T., and J. M. Cornwall, Electrostatic model of a quiet auroral arc, J. Geophys. Res., 85, 543, 1980.

Chiu, Y. T., and M. Schulz, Self-consistent particle and parallel electric field distributions in the magnetospheric-ionospheric auroral region, J. Geophys. Res., 83, 629, 1978.

Chiu, Y. T., A. L. Neuman, and J. M. Cornwall, On the structures and mappings of auroral electrostatic particles, J. Geophys. Res., $86,10,029,1981$.

Chiu, Y. T., J. M. Cornwall, J. F. Fennell, D. J. Gorney, and P. F. Mizera, Auroral plasmas in the evening sector: Satellite observations and theoretical interpretations, Space Sci. Rev., 35, 211, 1982.

Cornwall, J. M., The connection between radiation belt and auroral processes, Space Sci. Rev., 34, 235, 1983.

Cornwall, J. M., Exact solutions and low-frequency instability of the adiabatic auroral arc model, J. Geophys. Res., 93, 11,429, 1988 .
Cornwall, J. M., Low-frequency ionization-driven instability of a discrete auroral arc model, J. Geophys. Res., 95, 19,079, 1990.

Erikson, G. M., and R. A. Wolf, Is steady convection possible in the Earth's magnetotail?, Geophys. Res. Lett., 7, 897, 1980.

Evans, D. S., Precipitating electrons formed by a magnetic fieldaligned potential difference, J. Geophys. Res., 79, 2853, 1974.

Fejer, J. A., Theory of the geomagnetic daily disturbance variations, J. Geophys. Res., 69, 123, 1964.

Huang, C. Y., C. K. Goertz, L. A. Frank, and G. Rostoker, Observational determination of the adiabatic index in the quiet time plasma sheet, Geophys. Res. Lett., 16, 563, 1989.

Keskinen, M. J., P. K. Chaturvedi, and S. L. Ossakow, A low altitude mechanism for mesoscale dynamics, structure, and current filamentation in the discrete aurora, Geophys. Res. Lett., 19, $2199,1992$.

Kivelson, M. G., and H. E. Spence, On the possibility of quasistatic convection in the quiet magnetotail, Geophys. Res. Lett., 15, 1541, 1988.

Lindqvist, P.-A., and G. T. Marklund, A statistical study of highaltitude electric fields measured on the viking satellite, $J$. Geophys. Res., 95, 5867, 1990.

Lyons, L. R., Generation of large-scale regions of auroral currents, electric potentials, and precipitation by the divergence of the convection electric field, J. Geophys. Res., 85, 17, 1980.

Lyons, L. R., Discrete aurora as the direct result of an inferred high-altitude generating potential distribution, J. Geophys. Res., $86,1,1981$.

Mizera, P. F., and J. F. Fennell, Signatures of electric fields from high and low altitude particle distributions, Geophys. Res. Lett., 4, 311, 1977.

Reiff, P., H. Collin, J. Craven, J. Burch, J. Winningham, E. Shelley, L. Frank, and M. Friedman, Determination of auroral electrostatic potentials using high-and low-altitude particle distributions, J. Geophys. Res., 93, 7441, 1988.

Schield, M. A., J. W. Freeman, and A. J. Dessler, A source of field-aligned currents at auroral latitudes, J. Geophys. Res., 74, $247,1969$.

Schindler, K., and J. Bim, Self-consistent theory of time-dependent convection in the Earth's magnetotail, J. Geophys. Res., 87, 2263, 1982.

Spence, H. E., and M. G. Kivelson, The variation of the plasma sheet polytropic index along the midnight meridian in a finite width magnetotail, Geophys. Res. Lett., 17, 591, 1990.

Spence, H. E., M. G. Kivelson, and R. J. Walker, Static magnetic field models consistent with nearly isotropic plasma pressure, Geophys. Res. Lett., 14, 872, 1987.

Vasyliunas, V. M., Mathematical models of magnetospheric convection and its coupling to the ionosphere, in Particles and Fields in the Magnetosphere, edited by B. M. McCormac, p. 60, D. Reidel, Norwell, Mass., 1970.

Weimer, D. R., J. R. Kan, and S.-J. Akasofu, Variations of the polar cap potential measured during magnetic substorms, J. Geophys. Res., 97, 3945, 1992.

Whittaker, E. T., and G. N. Watson, A Course of Modern Analysis, Cambridge University Press, New York, 1952.

Wolf, R. A., Effects of ionospheric conductivity on convective flow of plasma in the magnetosphere, J. Geophys. Res., 75, 4677, 1970.

Wolf, R. A., R. W. Spiro, and F. J. Rich, Extension of convection modeling into the high-altitude ionosphere: Some theoretical difficulties, J. Atmos. Terr. Phys., 53, 817, 1991.

J. M. Cornwail, Department of Physics, University of California, 405 Hilgard Avenue, Los Angeles, CA 90024-1547.

(Received February 18, 1993; revised April 19, 1993; accepted April 19, 1993.) 


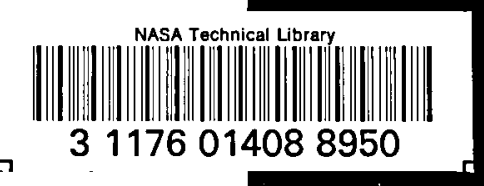

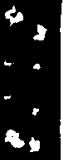

?

(A) THE AEROSPACE CORPORATION

2350 E. El Segundo Boulevard

EI Segundo, California 90245-4691 U.S.A. 